\title{
EXTRACTION AND SHAPE RECONSTRUCTION OF GUARDRAILS USING MOBILE MAPPING DATA
}

\author{
Hiroki Matsumoto, Yuma Mori, Hiroshi Masuda \\ The University of Electro-Communication, 1-5-1 Chofugaoka, Chofu, Tokyo 182-8585, Japan - \\ m1832102@edu.cc.uec.ac.jp, (yuma.mori, h.masuda)@uec.ac.jp
}

\section{Commission VI, WG VI/4}

KEY WORDS: MMS, Point-Cloud, Guardrail, Shape Reconstruction, Classification, Convolutional Neural Network

\begin{abstract}
:
The mobile mapping system (MMS) can acquire dense point-clouds of roads and roadside features. Roads are often separated into roadways and walkways in many urban areas. Since guardrails are installed to separate roadways and sidewalks, it is important to detect guardrails from point-clouds and reconstruct their 3D models for 3D street maps. Since there are a large variety of designs for guardrails in Japan, flexible methods are required for detection and reconstruction of guardrails. In this paper, we propose a new method for extracting guardrails from point-clouds, and reconstructing their 3D models. Since the MMS captures point-clouds and camera images synchronously, guardrails are detected using both point-clouds and images. In our method, point-clouds are segmented into small segments, and corresponding images are cropped from camera images. Then cropped images are classified into two classes of guardrails and others using the convolutional neural network. When guardrail points are obtained, 3D models of guardrails are reconstructed. However, point-clouds of guardrails are too sparse to reconstruct 3D shapes when guardrails consist of thin pipes. Since the same unit shape repeatedly appears in a guardrail, we create dense point-clouds by superimposing points of unit shapes. Then we reconstruct 3D shapes of pipes, beams, and poles of guardrails. In our evaluation using point-clouds in urban areas, our method could achieve good results of extraction and shape reconstruction of guardrails.
\end{abstract}

\section{INTRODUCTION}

The mobile mapping system (MMS) can acquire dense pointclouds of roads and roadside features. As shown in Figure 1, the MMS consists of laser scanners, digital cameras, GPSs and an IMU on the vehicle. The MMS allows us to capture dense pointclouds and sequential camera images while driving. By analysing point-clouds and camera images, we can obtain rich 3D information of roads and roadside features.

In this paper, we discuss methods to detect guardrails and reconstruct their 3D models, because guardrails are often installed to separate roadways and sidewalks. For generating faithful 3D street maps, it is important to detect guardrails from point-clouds and reconstruct their 3D models.

Many researchers developed methods for detecting roadside features from MMS data. Roadside features can be detected using geometric properties suitable for each object class. Various geometric properties have been investigated based on pointclouds (Yokoyama, et al., 2011, Masuda, et al., 2013, Aijazi, et al., 2013, Cabo, et al., 2014, Yang, et al., 2015, Aijazi, et al., 2013). Other researchers have studied supervised machine learning for automatically selecting useful geometric features (Golovinskiy, et al., 2009, Zhu, et al., 2010, Puttonen, et al., 2011, Ishikawa, et al., 2012, Lai, et al., 2009, Fukano, et al., 2015). However, these methods can recognize only limited classes of objects, and they did not handle guardrails.

For reconstructing 3D models, domain-specific knowledge is often used, because the MMS can capture only partial pointclouds of objects. So far, domain-specific knowledge has been introduced for symmetric objects (Pauly, et al., 2008), urban buildings (Nan, et al., 2010), houses (Lin, et al., 2013), and trees

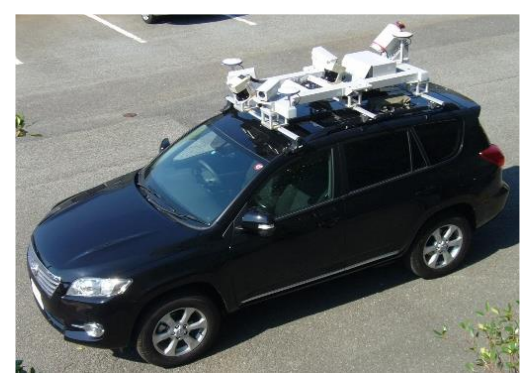

Figure 1. Mobile mapping system
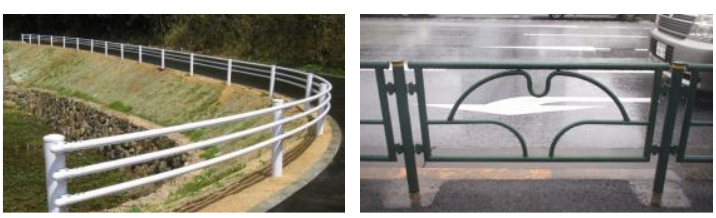

(a) Guardrail consisting of pipes

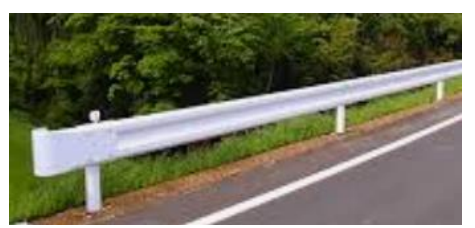

(b) Guardrail consisting of beams

Figure 2. Various types of guardrails

\footnotetext{
* Corresponding author
} 
(Livny, et al., 2010). He, et al. detected roadways and sidewalks by detecting road curbs (Fukano, et al., 2015, He, et al., 2012), but they did not detect walkways separated by guardrails.

For detecting and reconstructing guardrail shapes, flexible methods are required, because there are a large variety of designs for guardrails in Japan, as shown in Figure 2. Although the installation standard was determined by the Ministry of Land, but the shapes of guardrails were not specified in the standard. Therefore, many municipalities introduced unique shapes of guardrails in Japanese urban areas. In addition, it is required to process incomplete point-clouds for reconstructing 3D models of guardrails, because many guardrails consist of thin pipes and points on pipes are very sparse.

In this paper, we propose a new method for extracting and reconstructing guardrails using point-clouds and camera images. In our method, candidates of guardrails are extracted from pointclouds, and corresponding images are cropped from camera images. Then it is verified whether cropped images are guardrails or not. Then points of guardrails are extracted from point-clouds and the 3D models are created using guardrail points.

\section{OVERVIEW}

Figure 2 shows two typical types of guardrails. One group consist of pipes. They are often used in urban areas and sometimes represent symbols of municipalities. The other group of guardrails consist of beams, as shown in Figure 2(b). In this paper, we discuss these two types of guardrails.

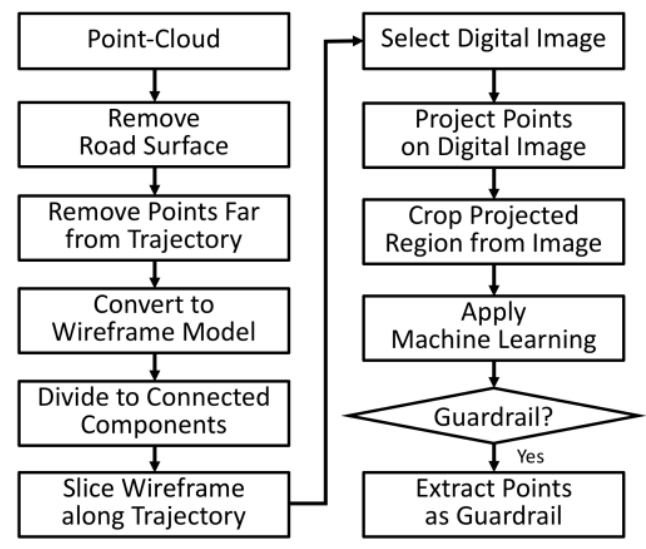

Figure 3. Process of extracting guardrail points

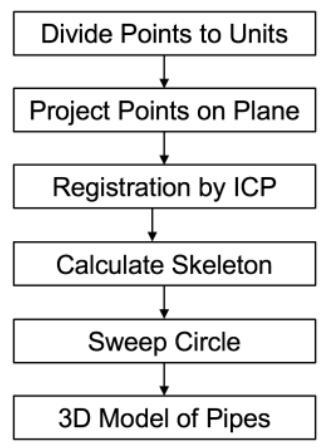

(a) Guardrail made of pipes

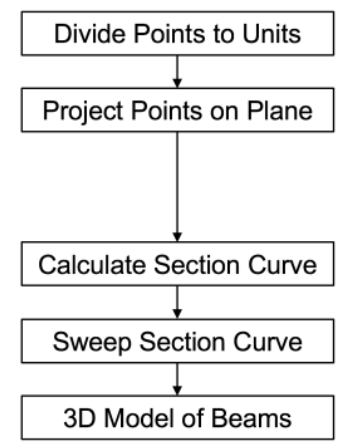

(b) Guardrail made of beams
The MMS used in this research is the Mitsubishi MMS-X (Mitsubishi Electric, 2018), as shown in Figure 1. The laser scanner is RIEGL VQ 250. The rotational frequency is $100 \mathrm{~Hz}$, and the scanning speed is 300,000 measurements per second. Point-clouds and camera images are captured while driving, and each point and image has GPS time.

The MMS captures point-clouds and images synchronously. Since the relative positions between the laser scanner and the cameras are fixed on the MMS, 3D points acquired with the laser scanner can be projected on 2D images using the well-known pinhole camera model (Weng, et al., 1992).

Figure 3 shows the process of extracting 3D points of guardrails, and Figure 4 shows the process of reconstructing 3D shapes of guardrails. For detecting guardrails, we use the method proposed by Mori, et al (Mori, et al., 2019). In this method, point-clouds are segmented into small segments, and points in each segment are projected onto a camera image. Then the projected area is cropped from the image. The cropped images are used for detecting guardrails using machine learning.

After guardrail points are obtained, 3D models of guardrails are reconstructed. However, point-clouds of guardrails are too sparse to reconstruct complete 3D shapes. Since the guardrail is made by connecting many unit shapes, we create dense point-clouds by subdividing guardrail points into unit shapes and superimposing points of the unit shapes. 3D models are created using superimposed dense point-clouds.

For reconstructing pipes in Figure 2(a), points of a unit shape are projected on a $2 \mathrm{D}$ plane. Then, skeletons of poles and pipes of guardrails are calculated from projected points. The radius of a pipe is estimated using the distances of points from the skeleton lines. The 3D model of each pipe is reconstructed by sweeping a circle along the skeleton.

For reconstructing beams of guardrails in Figure 2(b), points on a beam are projected on the 2D plane that are perpendicular to the ground and the guardrail plane. The projected points represent the section curve of the beam. The $3 \mathrm{D}$ shape of a beam is reconstructed by sweeping the section curve.

\section{EXTRACTION OF GUARDRAILS}

\subsection{Generation of Sliced Segments from Point-Clouds}

First, road surfaces are detected from point-clouds and points on roads are eliminated. Since the $\mathrm{z}$ values of roads can be estimated using the trajectory data of the MMS, points are removed when the $\mathrm{z}$ values are equal to or smaller than the estimated road height and the normal vectors calculated using neighbor points are close to the $\mathrm{Z}$ axis. In addition, since the guardrail is installed along roadways, points away from road surfaces are also removed.

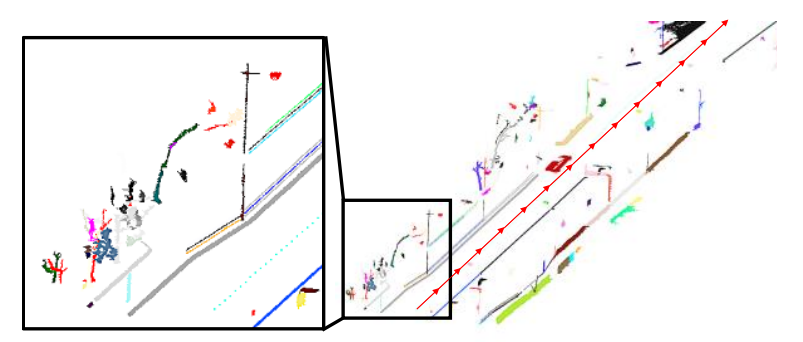

Figure 5. Extraction of roadside features

Figure 4. Process of shape reconstruction 
Point-clouds acquired by the MMS can be mapped on the 2D lattice using the rotation numbers and phase numbers, which are calculated using GPS time and scanner parameters (Kohira, et al., 2017). Therefore, we map point-clouds on the $2 \mathrm{D}$ lattice and convert them into a wireframe model by connecting neighbor points on the $2 \mathrm{D}$ lattice. Then, the wireframe model is subdivided into connected components. Figure 5 shows connected components, which include utility poles, traffic signals, road signs, trees, buildings, guardrails, and so on. A single component may contain multiple features.

Since guardrails have various lengths and may be curved, connected components are sliced into small segments using section planes perpendicular to the trajectory of the vehicle, as shown in Figure 6(a). In our method, each connected component is subdivided at the interval of $0.5 \mathrm{~m}$. Figure 6(b) shows sliced segments of a guardrail.

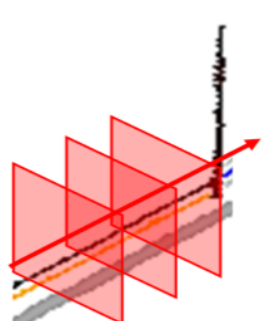

(a) Section planes

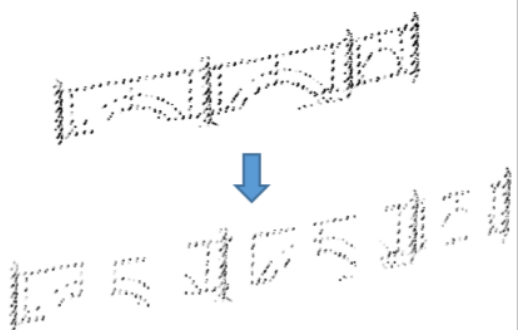

(b) subdivision of connected component
Figure 6. Subdivision along the vehicle trajectory

\subsection{Extraction of Cropped Images}

MMS synchronously captures 3D points and 2D images. Each point and each image have time stamps called GPS time, which is determined based on the signal from the satellite. By comparing GPS time, camera images corresponding to each sliced segment can be obtained.

Points in a sliced segment can be projected onto a $2 \mathrm{D}$ image. The relative position between the laser scanner and the digital camera is fixed and the trajectory of the laser scanner is stored as a series of positions and attitudes. Camera parameters can be also calculated using camera calibration algorithms. Therefore, 3D coordinates can be projected onto the image plane using a wellknown pin-hole camera model (Weng, et al., 1992), as shown in Figure 7.

When points are projected on an image, a rectangular region is generated so that all points are included. Then the rectangular region is cropped from the image. When multiple images correspond to the same sliced segment, the image that contains all projected points and has the largest rectangular area is selected. Figure 8 shows cropped images of sliced segments of a guardrail.

\subsection{Extraction of Guardrail points}

When cropped images are obtained, it is checked whether they are guardrails or not. We use Convolutional Neural Network (CNN) (Krizhevsky, et al., 2012) for two class classification. We introduce two classes of "guardrail" and "other object". In general, CNN needs a large number of training images for accurate classification. However, in our case, it is difficult to prepare a sufficient number of guardrail images. Therefore, we use a fine tuning method to train a classifier using a small number of images.

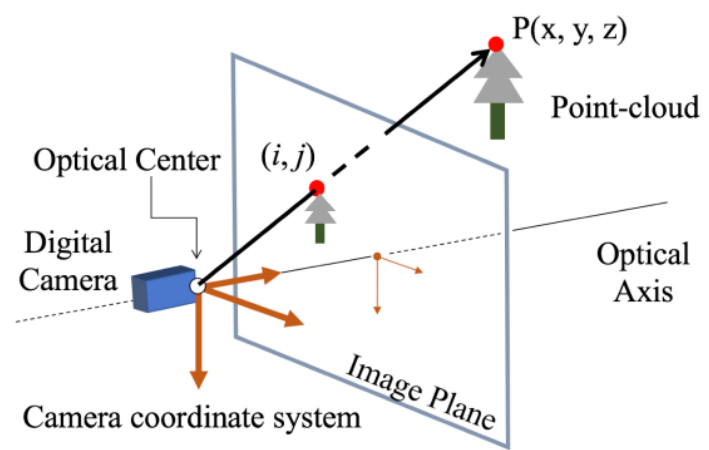

Figure 7. Projection of points onto an image plane

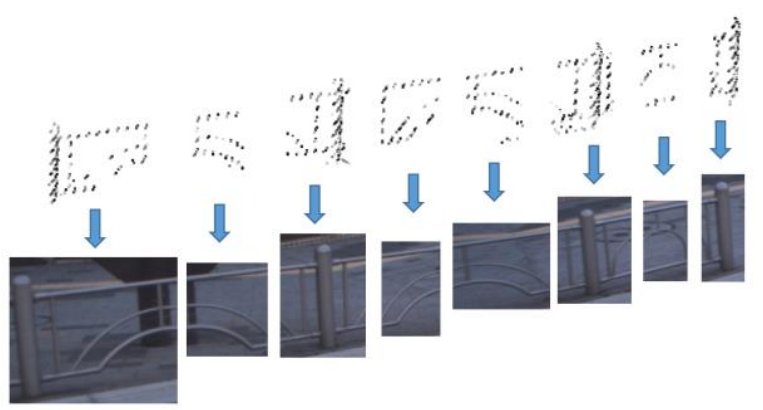

Figure 8. Cropped images of sliced segments
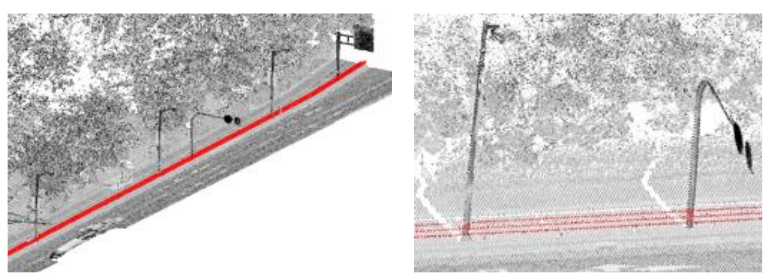

Figure 9. Extracted guardrail points

In fine tuning, neural network parameters are adjusted using a large number of images before learning specific classes.

In our method, the classifier is trained using cropped images with two class labels. Each cropped image is classified as "guardrail" or "other object". When an image is classified as "guardrail", the corresponding sliced segment is extracted as guardrail points.

However, images may be misclassified when there is an object whose shape is similar to a guardrail or an image contains only a small part of a guardrail. For correcting misclassified images, labels of sliced segments are modified according to adjacent sliced segments. When a sliced segment with the guardrail label is isolated, it is regarded as "other object". On the other hand, most consecutive sliced segments have guardrail labels, sliced segments with "other object" are regarded as guardrails. Figure 9 shows extracted guardrail points after correcting labels.

\section{SHAPE RECONSTRUCTION OF GUARDRAILS}

\subsection{Subdivision of Guardrail into Unit Shapes}

Generally, guardrails are made of basic units and poles. As shown in Figure 10(a), multiple basic units are assembled using poles installed on the ground. Figure 10(b) shows guardrail points. Since guardrail points are too sparse to reconstruct guardrail 
shapes, we increase the density of guardrail points by subdividing guardrail points into unit shapes and superimposing points of the unit shapes.

To subdivide guardrail points into unit shapes, poles are detected from guardrail points, as shown in Figure 10(c). We detect poles by using the method proposed by (Fukano, et al., 2015). Figure 11 shows the process of pole detection. First points are projected on the 2D lattice and converted into a wireframe model by connecting neighbor points on the 2D lattice (Figure 11(b)). Then

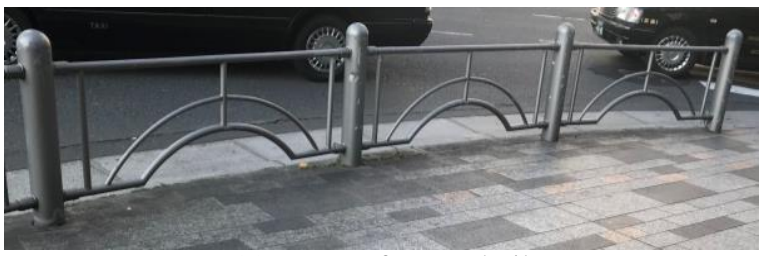

(a) Image of a guardrail

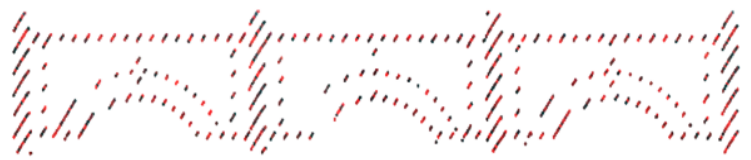

(b) Point-cloud

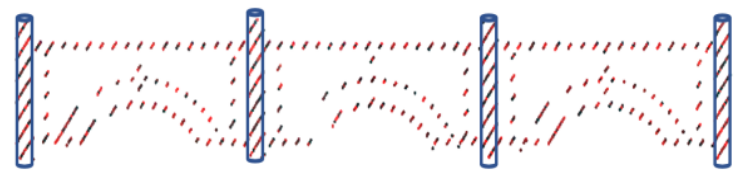

(c) Detection of poles

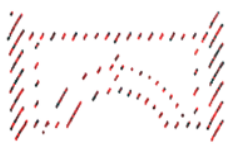

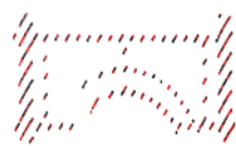

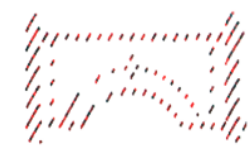

(d) Subdivision into unit shapes

Figure 10. Subdivision guardrail points into unit shapes

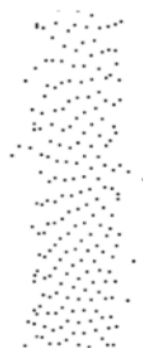

(a) Points

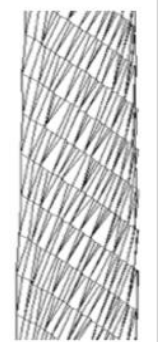

(b) Wireframe

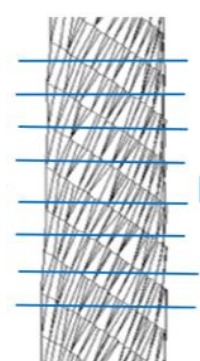

(c) Section planes

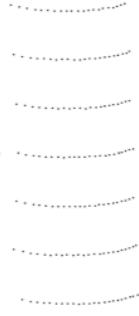

(d) Intersections

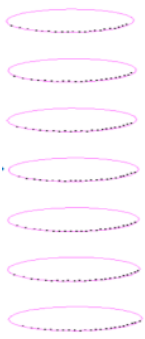

(e) Circle fitting

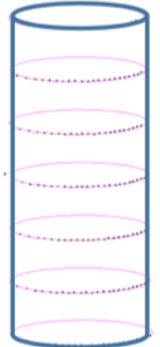

(f) Cylindrical pole
Figure 11. Process of pole detection the wireframe model is subdivided using horizontal section planes, as shown in Figure 11(c). Intersection points are obtained as shown in Figure 11(d), and the intersection points on each section plane are fitted to a circle, as shown in Figure 11(e). Finally, a cylindrical pole is generated by connecting vertically aligned circles. The height of the pole is determined using the ground and the highest point.

When poles are detected, guardrail points are subdivided into unit shapes so that each unit shape includes two poles, as shown in Figure 10(d).

\subsection{Superimposing Points of Unit Shapes}

For reconstructing thin pipes, it is necessary to increase the density of points. Therefore, we superimpose points of unit shapes, as shown in Figure 12.

First each unit shape is projected on the plane. The plane is calculated using the principal component analysis. The coordinate system is determined as shown in Figure 12. The zaxis is the direction of the plane normal, and the y-axis is determined as the direction of poles. The origin is the center of the rectangle region in which points are projected.

Points of unit shapes are superimposed on the same plane. Initially, the superimposed points of unit shapes are roughly registered. For precise registration, the iterative closest point (ICP) algorithm is applied points of each unit shape. Figure 12 shows points registered using ICP. For reliably reconstructing guardrail shapes, as many unit shapes as possible are superimposed.
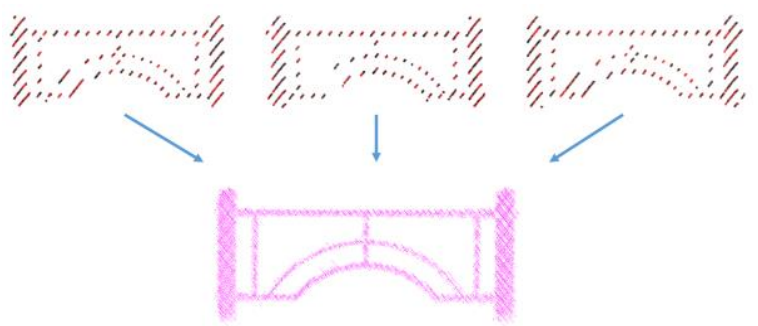

Figure 12. Superimposing points of unit shapes

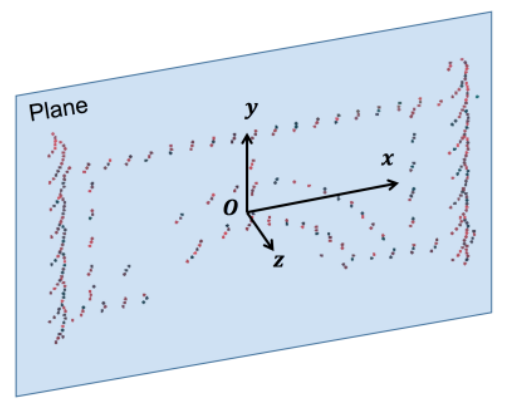

Figure 13. Coordinate system of unit shape

\subsection{Reconstruction of Guardrails Consisting of Pipes}

Point-clouds acquired by the MMS are very noisy. Since it is difficult to stably extract thin pipes using the pole detection 
method, we detect pipes in two steps. In the first step, vertical pipes are detected, and then other pipes are detected.

For detecting vertical pipes, a histogram is created in the direction of the $\mathrm{x}$ axis, as shown in Figure 14. When there are vertical pipes in guardrail points, peaks appear in the histogram. The peaks are detected by setting a threshold so that continuous regions are separated above the threshold. The diameter of each vertical pipe is determined so that a certain percentage of points are included in the region of the vertical pipe. Then the skeletons of vertical pipes are determined at the center of the pipe region, as shown in Figure 15.

In the next step, non-vertical pipes are detected. The process is shown in Figure 16. When vertical pipes are detected, their points are removed from guardrail points, as shown in Figure 16(a). Then remaining points are divided at equal intervals in the $\mathrm{x}$ axis direction, as shown in Figure 16(b). In this research, we set $30 \mathrm{~mm}$ as the interval. Then divided points are converted to a

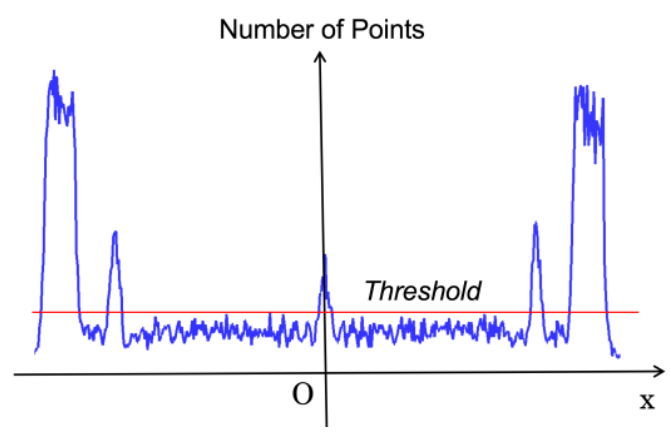

Figure 14. Histogram of points

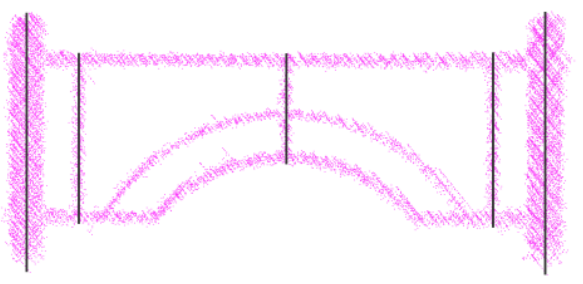

Figure 15. Skeletons of vertical pipes

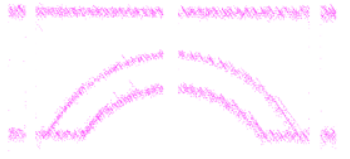

(a) Removing vertical pipes

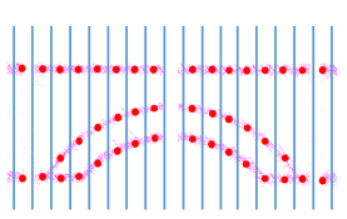

(c) Center points

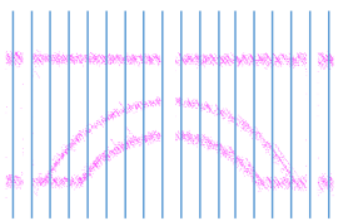

(b) Vertical regions

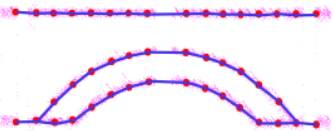

(d) Connection
Figure 16. Skeletons of horizontal and curved pipes wireframe model using the Delaunay triangulation, and the wireframe is divided to connected components. Then the center of each connected component is calculated as shown in Figure 16(c). Finally, neighbor points are connected and polylines are created, as shown in Figure 16(d).

Since created polylines are distorted, they are smoothed using a low-pass filter. We use the low-pass filter proposed by (Taubin, et al., 1995). When a polyline is approximately a straight line, it is fitted to a straight line. In addition, straight lines are almost horizontal, they are regarded as horizontal pipes.

In the next step, polylines are extended and connected. First, when the end point of a polyline is close to poles or vertical pipes, the polyline is extended and connected with the pole or the vertical pipe. When the end points of two polylines are closely located, the two polylines are extended and the intersection point is calculated.

Finally, cylindrical pipes are generated by sweeping circles along polylines. Vertical pipes are also generated by sweeping circles vertically. As a result, a 3D model of a unit shape is created. In our method, a guardrail is divided into unit shapes. For reconstructing the overall shape of a guardrail, 3D models of the unit shape are copied between poles.

\subsection{Reconstruction of Guardrails Consisting of Beams}

Figure 17 shows a guardrail consisting of beams. The beams are manufactured by bending a long iron plate. For reconstructing beam shapes, section curves of beams are extracted.

First, poles are detected using the pole detection method. Then, each guardrail is subdivided into unit shapes. In cases of beams, a relatively large rectangle region is filled by points. Therefore, beams can be distinguished from pipes.

To calculate a section curve, the coordinate system is defined as shown in Figure 18. Then points on poles are eliminate and the

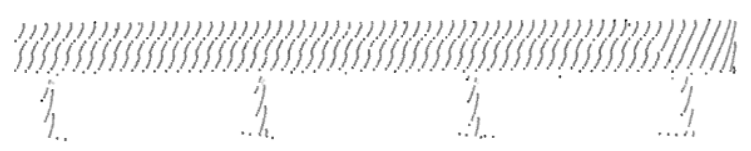

Figure 17. Point-cloud of a guardrail consisting of beams

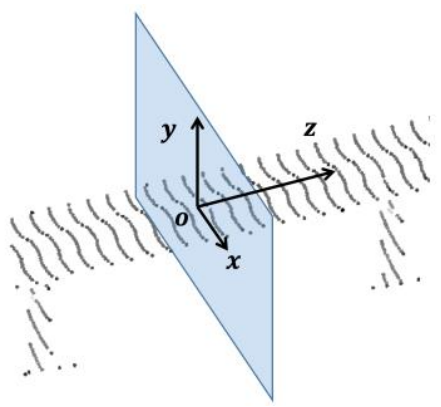

Figure 18. Projection plane 
remaining points are projected on the x-y plane. Figure(a) shows projected points.

Section curves are created in the same way of pipe reconstruction. First, points are segmented using horizontal lines, as shown in Figure 19(b), and the center point is calculated in each region, as shown in Figure 19(c). The interval of horizontal lines is specified as $10 \mathrm{~mm}$. Then the section curve is obtained as a polyline, as shown in Figure 19(d). The polyline is smoothed using the low-pass filter. Finally, a 3D model of the beam shape is reconstructed by sweeping the section curve along the direction of the guardrail.

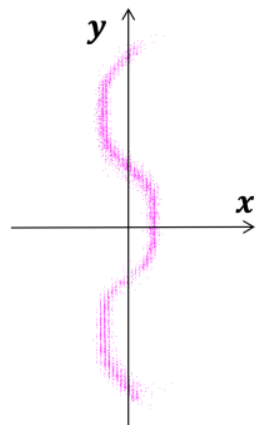

(a) Projected points

(b) Horizontal regions

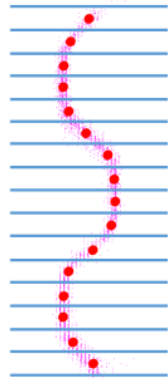

(c) Center points

(d) Polyline of section curve

Figure 19. Skeleton of beam shape

\section{EXPERIMENTAL RESULTS}

\subsection{Detection of Guardrail Points}

We evaluated our method using point-clouds and camera images captured in urban areas in Japan. First we extracted guardrails from the MMS data. We generated about 10,000 sliced pointclouds and cropped images from the MMS data. For obtaining the ground-truth, we manually added a label "guardrail" or "other object" to each cropped image.

Table 1. The number of sliced segments

\begin{tabular}{|c|c|c|}
\hline & Training data & Test data \\
\hline guardrail & 3912 & 777 \\
\hline other object & 3910 & 1910 \\
\hline
\end{tabular}

Table 2. Classification results

\begin{tabular}{|c|c|c|c|}
\hline & Recall & Precision & F-Measure \\
\hline guardrail & $97.9 \%$ & $91.5 \%$ & $94.6 \%$ \\
\hline other object & $94.6 \%$ & $98.7 \%$ & $96.6 \%$ \\
\hline
\end{tabular}
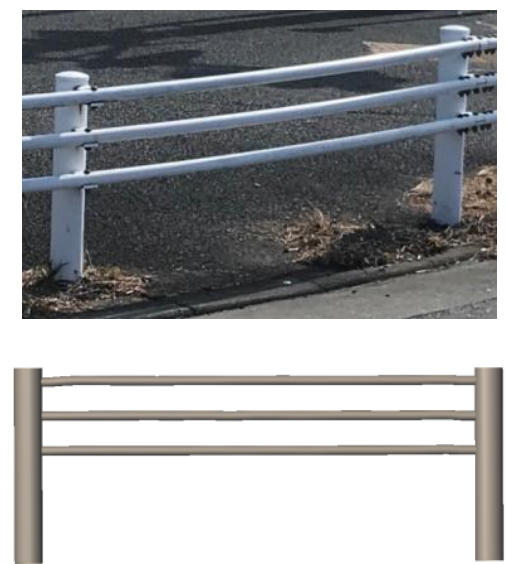

(a) Simple pipes
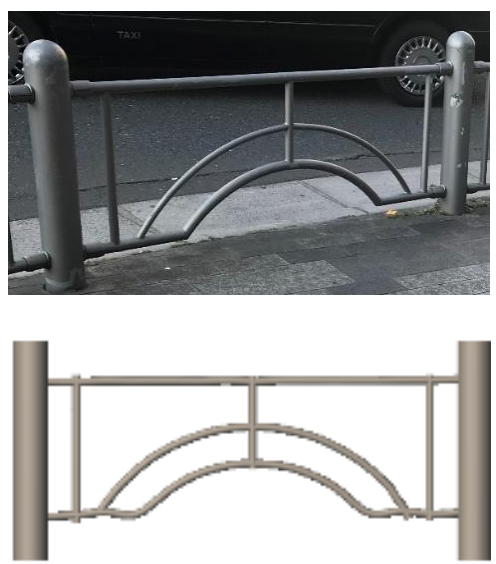

(b) Complicated pipes

Figure 20. Unit shapes of guardrails consisting of pipes

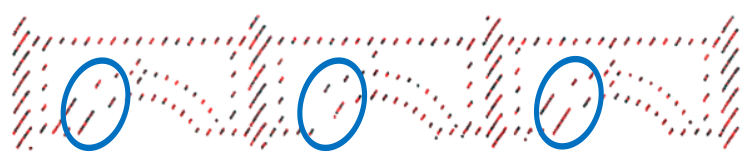

Figure 21. Pipes on which points are sparse
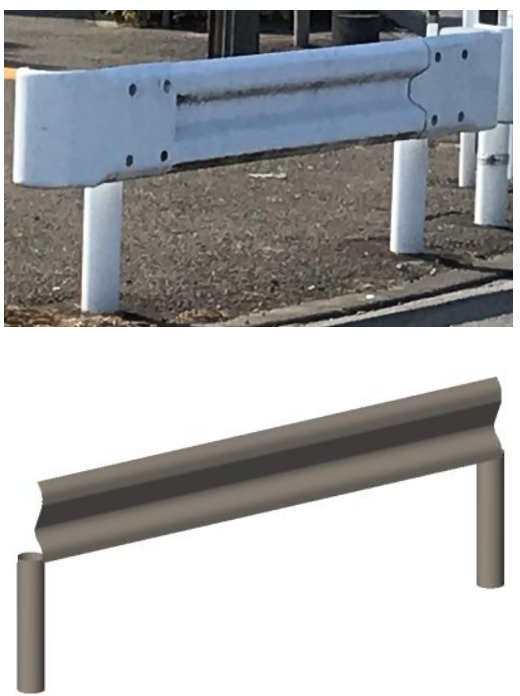

Figure 22. Unit shape of guardrail consisting of beams 
For evaluation, we divided the MMS data into training data and test data. Table 1 shows the numbers of images in the training data and the test data.

Table 2 shows the classification results using $\mathrm{CNN}$ and label correction. Our method achieved very good success rates, and could detect almost all guardrails. However, only a few guardrails could not be detected. This is because the lengths of guardrails were very short and the unit shapes were not regular sizes.

\subsection{Reconstruction of Guardrails}

We reconstructed guardrail shapes using detected guardrail points. Figure 20 shows reconstructed unit shapes of guardrails consisting of pipes. In both cases, adequate 3D models could be generated.

In our method, the quality of reconstructed shapes depends on the numbers of superimposed unit shapes. If the number of unit shapes is small, all pipes may not be reconstructed.

We investigated the numbers of unit shapes that were required to reconstruct all pipes. In the case of Figure 20(a), 4 or more unit shapes were required. On the other hand, in the case of Figure 20(b), 25 or more unit shapes were required to reconstruct all pipes. This is because the diagonal pipes shown in Figure 21 were almost parallel to the direction of scan lines of the laser scanner, and therefore, the point density was very low. To reconstruct these pipes, many unit shapes were required.

We also reconstructed guardrails consisting of beams. Figure 22 shows a reconstructed unit shape. We investigated the number of superimposed unit shapes. In this case, only one unit shape was enough to reconstruct the beam shape, because points on a beam were much denser than points on a pipe.

\section{CONCLUSION}

In this paper, we proposed a method for detecting guardrails from the MMS data. We also proposed a method for reconstructing guardrail shapes using the detected guardrail points. In our method, point-clouds were segmented into small groups of points, and images corresponding with each group was cropped from original camera images. Cropped images were classified using CNN into "guardrail" or "other object". Then classified labels were corrected using adjacency of segmented points. For reconstructing 3D shapes of guardrails, guardrail points were segmented into unit shapes, and points of unit shapes were superimposed to generate dense point-clouds. Then the 3D shape of each unit shape was reconstructing by extracting skeletons of pipes and section curves of beams. We evaluated our methods using point-clouds of urban areas in Japan. In our experiments, guardrail points could be extracted with sufficiently good success rates, and $3 \mathrm{D}$ shapes of three types of unit shapes could be successfully reconstructed.

In the future work, we would like to evaluate our method using point-clouds captured in many restricts, because there are only one or two types of guardrails in each restrict. We would also like to implement codes for export guardrail shapes to actual 3D maps.

\section{ACKNOWLEDGEMENTS}

MMS data in this paper are courtesy of AISAN Technology Co.Ltd. and Sanei-Giken Co. We would like to thank for their helpful support.

\section{REFERENCES}

Yokoyama, H., Date, H., Kanai, S., \& Takeda, H. (2011). Polelike objects recognition from mobile laser scanning data using smoothing and principal component analysis. Int. Arch. Photogramm. Remote Sens. Spat. Inf. Sci, 38, 115-120.

Masuda, H., Oguri, S., \& He, J. (2013, January). Shape reconstruction of poles and plates from vehicle-based laser scanning data. In Informational Symposium on Mobile Mapping Technology (Vol. 201).

Aijazi, A., Checchin, P., \& Trassoudaine, L. (2013). Segmentation based classification of 3D urban point clouds: A super-voxel based approach with evaluation. Remote Sensing, 5(4), 1624-1650.

Cabo, C., Ordoñez, C., García-Cortés, S., \& Martínez, J. (2014). An algorithm for automatic detection of pole-like street furniture objects from Mobile Laser Scanner point clouds. ISPRS Journal of Photogrammetry and Remote Sensing, 87, 4756.

Yang, B., Dong, Z., Zhao, G., \& Dai, W. (2015). Hierarchical extraction of urban objects from mobile laser scanning data. ISPRS Journal of Photogrammetry and Remote Sensing, 99, 4557.

Aijazi, A., Checchin, P., \& Trassoudaine, L. (2013). Segmentation based classification of 3D urban point clouds: A super-voxel based approach with evaluation. Remote Sensing, $5(4), 1624-1650$.

Golovinskiy, A., Kim, V. G., \& Funkhouser, T. (2009, September). Shape-based recognition of 3D point clouds in urban environments. In 2009 IEEE 12th International Conference on Computer Vision (pp. 2154-2161). IEEE.

Zhu, X., Zhao, H., Liu, Y., \& Zha, H. (2010). Segmentation and classification of range image from an intelligent vehicle in urban environment. IEEERSJ International Conference, 18-22.

Puttonen, E., Jaakkola, A., Litkey, P., \& Hyyppä, J. (2011). Tree classification with fused mobile laser scanning and hyperspectral data. Sensors, 11(5), 5158-5182.

Ishikawa, K., Tonomura, F., Amano, Y., \& Hashizume, T. (2012). Recognition of road objects from Mobile Mapping data, Asian Conference on Design and Digital Engineering.

Lai, K., \& Fox, D. (2009, June). 3D laser scan classification using web data and domain adaptation. In Robotics: Science and Systems (Vol. 2)

Fukano, K., \& Masuda, H. (2015). DETECTION AND CLASSIFICATION OF POLE-LIKE OBJECTS FROM MOBILE MAPPING DATA. ISPRS Annals of Photogrammetry, Remote Sensing \& Spatial Information Sciences, 2. 
Pauly, M., Mitra, N. J., Wallner, J., Pottmann, H., \& Guibas, L. J. (2008, August). Discovering structural regularity in 3D geometry. In ACM transactions on graphics (TOG) (Vol. 27, No. 3, p. 43). ACM.

Nan, L., Sharf, A., Zhang, H., Cohen-Or, D., \& Chen, B. (2010, July). Smartboxes for interactive urban reconstruction. In ACM Transactions on Graphics (TOG) (Vol. 29, No. 4, p. 93). ACM.

Lin, H., Gao, J., Zhou, Y., Lu, G., Ye, M., Zhang, C., ... \& Yang, R. (2013). Semantic decomposition and reconstruction of residential scenes from LiDAR data. ACM Transactions on Graphics (TOG), 32(4), 66.

Livny, Y., Yan, F., Olson, M., Chen, B., Zhang, H., \& El-Sana, J. (2010). Automatic reconstruction of tree skeletal structures from point clouds. ACM Transactions on Graphics (TOG), 29(6), 151.

He, J., \& Masuda, H. (2012). Reconstruction of roadways and walkways using point-clouds from mobile mapping system. In Asian Conference on Design and Digital Engineering.

Weng, J., Cohen, P., \& Herniou, M. (1992). Camera calibration with distortion models and accuracy evaluation. IEEE Transactions on Pattern Analysis \& Machine Intelligence, (10), 965-980.

Mori, Y., Masuda, H., 2019. Extraction of Guardrails on Roads Using Point Clouds and Images from Mobile Mapping Systems, ACDDE Asian Conference on Design and Digital Engissneering.

Kohira, K., \& Masuda, H. (2017). POINT-CLOUD COMPRESSION FOR VEHICLE-BASED MOBILE MAPPING SYSTEMS USING PORTABLE NETWORK GRAPHICS. ISPRS Annals of Photogrammetry, Remote Sensing \& Spatial Information Sciences, 4.

Krizhevsky, A., Sutskever, I., \& Hinton, G. E. (2012). Imagenet classification with deep convolutional neural networks. In Advances in neural information processing systems (pp. 10971105)

Taubin, G. (1995, September). A signal processing approach to fair surface design. In Proceedings of the 22nd annual conference on Computer graphics and interactive techniques (pp. 351-358). ACM. 